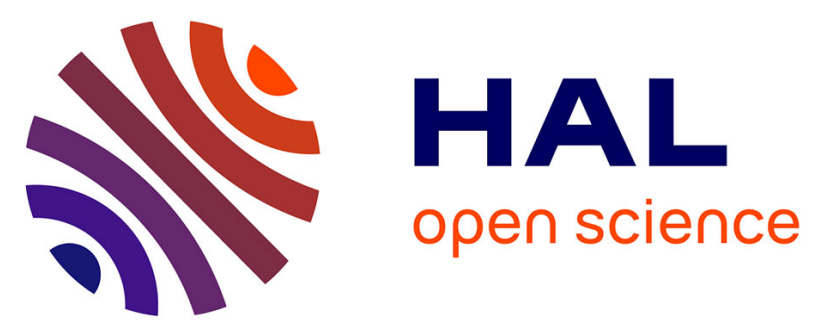

\title{
Simulation study of an X-ray diffraction system for breast tumor detection
}

Fanny Marticke, G Montémont, C Paulus, Olivier J.J. Michel, Jerome I. Mars, L I Verger

\section{- To cite this version:}

Fanny Marticke, G Montémont, C Paulus, Olivier J.J. Michel, Jerome I. Mars, et al.. Simulation study of an X-ray diffraction system for breast tumor detection. Nuclear Instruments and Methods in Physics Research Section A: Accelerators, Spectrometers, Detectors and Associated Equipment, 2017, 867, pp.20 - 31. 10.1016/j.nima.2017.04.026 . hal-01552429

\section{HAL Id: hal-01552429 \\ https://hal.science/hal-01552429}

Submitted on 24 Aug 2017

HAL is a multi-disciplinary open access archive for the deposit and dissemination of scientific research documents, whether they are published or not. The documents may come from teaching and research institutions in France or abroad, or from public or private research centers.
L'archive ouverte pluridisciplinaire HAL, est destinée au dépôt et à la diffusion de documents scientifiques de niveau recherche, publiés ou non, émanant des établissements d'enseignement et de recherche français ou étrangers, des laboratoires publics ou privés. 


\title{
Simulation study of an X-ray diffraction system for breast tumor detection
}

\author{
F. Marticke ${ }^{\mathrm{a}, \mathrm{b}}$, G. Montemont ${ }^{\mathrm{a}}$, C. Paulus ${ }^{\mathrm{a}}$, O. Michel ${ }^{\mathrm{b}}$, J. I. Mars ${ }^{\mathrm{b}}$, \\ L. Verger ${ }^{a}$ \\ ${ }^{a}$ Univ. Grenoble-Alpes, F-38000 Grenoble, France \\ CEA, LETI, Minatec Campus, F-38054 Grenoble, France \\ ${ }^{b}$ Univ. Grenoble-Alpes, Gipsa-Lab, F-38000 Grenoble, France \\ CNRS, Gipsa-Lab, F-38000 Grenoble, France
}

\begin{abstract}
X-ray diffraction (XRD) is a powerful technique used to determine the molecular structure of biological tissues. In breast tissues for example, the scattering signatures of dense fibroglandular tissue and carcinoma have been shown to be significantly different. In this study, XRD was used as a second control level when conventional mammography results were unclear, for instance because of overly high breast density. A system optimized for this issue, called multifocal XRD, was developed combining energy dispersive spectral information at different scattering angles. This system allows depth-imaging in one go but needs an $\mathrm{x}, \mathrm{y}$-direction scan to image the region conventional mammography identified as suspect. The scan-time for about $10 \mathrm{~cm}^{3}$ with an incident flux of about $4.8 \cdot 10^{7}$ photons per second would be around 2 seconds. For this study, breast phantoms with and without cancerous nodule were simulated to assess the separation power of the method and to determine the radiation dose required to obtain nearly ideal separation. For tumors situated in the center of the breast, the required dose was only about $0.3 \mathrm{mGy}$, even for breasts with high density. The tumor position was shown to have a low impact on detectability provided it remained in a zone where the system was sufficiently sensitive. The influence of incident spectrum maximum energy was also studied. The required dose remained very low with any of the incident spectra tested. Finally, an image slice was reconstructed in the $\mathrm{x}$-direction and showed that the system can detect the
\end{abstract}

Email address: fanny.marticke@gmail.com (F. Marticke) Preprint submitted to Nuclear Instruments and Methods in Physics Research Section March 19, 2017 
presence of a small tumor $(4 \mathrm{~mm})$. Hence, XRD is a very promising tool to reduce the number of unnecessary invasive breast biopsies.

Keywords: X-ray scattering, breast cancer, breast phantom simulation, radiation dose, system optimization, depth imaging, reconstruction.

\section{Introduction}

Conventional mammography is based on the premise that normal breast tissue and cancerous tissue differ in how they absorb X-rays. Mammography is currently believed to be the most effective breast screening tool. However, it is limited by the low contrast between the details to be detected (e.g. nodules) [1] and the background composed of adipose and fibroglandular tissues. The higher the proportion of fibroglandular tissue (i.e., the more dense the breast tissue), the more difficult it will be to detect a small lesion. This difficulty can lead to false negative or false positive diagnoses, resulting in missed cancer detection or unnecessary biopsies.

As breast biopsy is an invasive technique which causes patients considerable stress, the number of unnecessary ones should be reduced. This article proposes a non-invasive X-ray diffraction (XRD) method which could reduce the number of unnecessary breast biopsies. XRD can detect the molecular structure of biological tissues [2] which is not possible with X-ray absorption. Hence, it delivers additional information about the tissue to be classified. The potential of XRD to improve breast cancer diagnosis was recognized by numerous authors. Some of them suggested to enhance contrast of conventional mammography by combining transmission and coherent scattering images [3-5]. XRD as a standalone technique was also considered. Different XRD computed tomography systems were proposed [6-9]. The most important problem of XRD in breast cancer diagnosis is the system sensitivity, which implies long measurement time [10-12] and high dose delivery. Chaparian et al. [13, 14] proposed a method to optimize an energy dispersive X-ray diffraction system for clinical applications in terms of sensitivity notably. Though, this system only acquires at one scattering 
angle and acquisition of thicker objects requires a depth scan. Moreover, their study did not include dose considerations. Ghammraoui et al. [15, 16] analyzed the impact of delivered dose on image quality in coherent scatter computed tomography (CSCT) of the breast. The present study does not use tomography but a system requiring only a simple $\mathrm{x}, \mathrm{y}$-scan to perform volumic XRD imaging. Its purpose was to determine the dose necessary to detect a $4 \mathrm{~mm}$ breast tumor using this XRD technique.

We propose an optimized XRD system for use as a second control after conventional mammography with suspicious outcome. Using a convergent collimation system and a spectroscopic imaging detector, the system combines energy dispersive X-ray diffraction (EDXRD) spectral information at different scattering angles. A multi-angle EDXRD approach was suggested by other research teams $[17,18]$ and it was shown that this technique allows an increase of system sensitivity as well as an increase in the accessible momentum transfer range $[19]$.

The aim of this study was to show that this system can detect a small tumor of about $4 \mathrm{~mm}$ in a fibroglandular tissue background while only requiring exposure to a reasonable (not more than in conventional mammography) dose of radiation. This feasibility was demonstrated using simulations of XRD spectra for breast phantoms with different tissue compositions (varying breast density, with or without carcinoma nodule). The ability of the system to distinguish between spectra with or without carcinoma was assessed, and the dose required to obtain faithful distinction between samples was determined. The impact of tumor position and the energy level of the incident spectrum on the required dose were also studied. Finally, one scan slice measurement in the x-direction was simulated and reconstructed to confirm assessed system performances. 


\subsection{X-ray diffraction}

Coherent X-ray scattering or Rayleigh X-ray scattering, leading to X-ray diffraction when analyzing crystalline samples, is commonly used to determine the molecular structure of matter. Classically, coherent scattering is explained by the interaction between the electric field associated with the X-ray beam and the distribution of the electron charge in the analyzed sample. Under the action of the incident electric field, electrons oscillate and emit radiation with the same energy as the incident X-ray photon, but in a different direction. Radiation emitted by different electrons in the same or different atoms can constructively interfere to produce a typical X-ray scattering pattern. These patterns depend on the variable $\chi$ defined as:

$$
\chi=\frac{E}{h c} \cdot \sin \left(\frac{\theta}{2}\right)
$$

$\chi$ is given in $\mathrm{nm}^{-1}$ and is proportional to the momentum transferred to the photon causing it to be scattered at an angle $\theta$. $E$ corresponds to the photon energy, $h$ to Planck's constant and $c$ to the speed of light $\left(1 / h c \approx 0.806 \mathrm{keV}^{-1} \cdot \mathrm{nm}^{-1}\right)$. Hereafter, we will refer to $\chi$ as the momentum transfer.

The differential cross-section per electron for each solid angle is given by:

$$
\frac{d \sigma_{c o h}}{d \Omega}=\frac{d \sigma_{T h}}{d \Omega} \cdot f_{I A M}^{2}(\chi, Z) \cdot s(\chi)
$$

where $\frac{d \sigma_{T h}}{d \Omega}=r_{e}^{2} \frac{1+\cos ^{2} \theta}{2}$ is the classical Thomson cross-section for a free electron, $f_{I A M}$ is the coherent scatter form factor for independent atoms, accounting for interference between X-rays scattered by different electrons from the same atom; $s$ is the molecular interference function accounting for intraand intermolecular interference. This function corresponds to discrete Bragg peaks for materials with well defined long range orders, such as crystals. In the case of amorphous materials presenting a short-range order, such as biological tissues, $s$ will be a continuous function with an oscillatory behavior around unit value, and a decreasing amplitude as $\chi$ tends toward higher values. 
According to equation (1), XRD spectra can be measured in two ways. Usually, XRD spectra are measured by acquiring the diffraction signal as a function of $\theta$ at a fixed beam energy. This technique is called angular dispersive X-ray diffraction (ADXRD). For their acquisition a monochromatic X-ray beam is necessary, which strongly reduces the photon flux if a conventional X-ray tube is used. The second technique, energy dispersive X-ray diffraction (EDXRD), requires X-ray spectroscopic detectors capable of measuring the scattered intensity at a fixed scattering angle $\theta$, across a range of energies. As a conventional polychromatic X-ray tube can be used for this method, there is no photon flux problem. In general, EDXRD spectra present a lower resolution than ADXRD as in addition to resolution factors that are common to both methods (e.g. pixel size, object voxel size, angular resolution of the system...) spectral resolution of the detector also degrades the resolution. In ADXRD, the spectral width of the incident spectrum impacts the resolution but this spectral width only exists around the chosen energy whereas in EDXRD each energy bin of the detector presents a given spectral width. Nevertheless, EDXRD presents certain advantages compared to ADXRD. First of all, as has already been mentioned, the use of a polychromatic conventional X-ray tube is possible. Furthermore, one detector pixel can acquire the entire XRD pattern of a given object voxel. Therefore, it is possible to acquire the XRD signatures of different object in one pass which significantly increases the acquisition speed. It is also possible to combine the classical ADXRD and EDXRD techniques in order to increase system sensitivity, which will be proposed in the following study.

\subsection{X-ray diffraction in breast imaging}

The diffraction patterns of biological tissues, which correspond to a molecular structure with short-range order, are characterized by one or more smooth peaks at well-defined momentum transfer values (Fig. 1). Several studies attempted to classify different breast tissues using EDXRD [9, 10, 12, 20, 21], or ADXRD [8, 22-25]. A common result of these studies is that healthy adipose tissue produces a sharp peak at low momentum transfer values around $1.1 \mathrm{~nm}^{-1}$, 
while carcinoma presents a broad peak at higher momentum transfer values around $1.6 \mathrm{~nm}^{-1}$ (Fig. 1). This higher value corresponds to the maximum of the water scattering signature. Though, the difference between the scattering signatures of cancerous tissue and benign tumor was found to be very small by Pani et al. [9] (Fig. 1.a), and it does not seem possible to distinguish these two tissue types by XRD. Kidane et al. [10] found fibroadenoma scattering signature to have a maximum around $1.6 \mathrm{~nm}^{-1}$ as carcinoma and with scattering intensity at lower momentum transfer values between fibroglandular and cancerous tissue (Fig. 1.b). This signature seems to be easier to separate from carcinoma than the pattern determined by Pani et al. but more difficult than fibroglandular tissue. The attenuation of carcinoma and benign tumors is very similar. However, their shape is different. A fibroadenoma is well defined and sharply demarcated, whereas a carcinoma is ill-defined and has a stellate form. Hence, using the anatomical information from classical mammography or breast CT, it might be possible to distinguish between benign and malignant neoplasm.

Therefore, in this study we concentrate on distinguishing between healthy fibroglandular tissue and carcinoma. Both tissue types are very dense and it is often difficult to distinguish between them using conventional mammography because of their very similar absorption coefficients. Their XRD signatures, in contrast, are quite different. Fig. 1.b shows scattering profiles for the two types of tissue, as determined by [10] on a statistically significant number of samples. We used these scattering profiles as reference signatures for this study.

The main difference between fibroglandular and cancerous tissues is situated at low momentum transfer values around the fat peak (around $1.1 \mathrm{~nm}^{-1}$ ), as shown in Fig. 1.b. The $\chi$-values of interest are between $1 \mathrm{~nm}^{-1}$ and $2.5 \mathrm{~nm}^{-1}$ in the present work. 


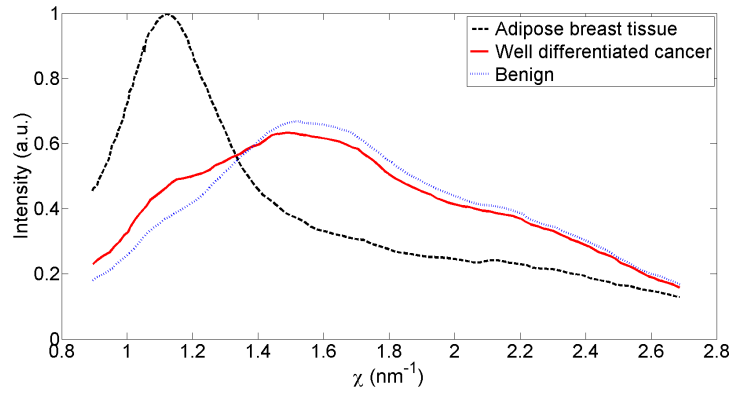

(a) Breast adipose tissue (black dashed line), cancerous tissue (red line) and benign tumor (blue broken line) [9].

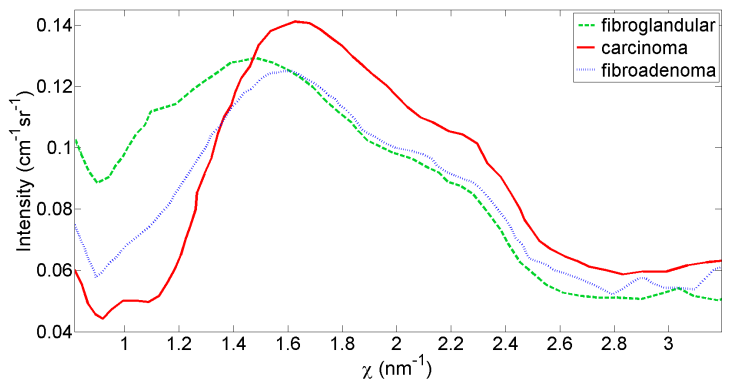

(b) Fibroglandular tissue (light green dashed line), pure carcinoma (red line) and fibroadenoma (blue broken line) [10].

Figure 1: Scattering signatures for different breast tissues. 


\section{Materials and methods}

\subsection{X-ray diffraction system}

As explained previously, XRD is proposed to be used as a second control when conventional X-ray mammography results do not give a clear diagnosis. Conventional mammography allows to determine the position of the suspect region in the $(\mathrm{x}, \mathrm{y})$ plane, but not its depth within the breast. Hence, the proposed XRD system must be able to target a specific region in the $(\mathrm{x}, \mathrm{y})$ plane and to image the whole breast thickness, i.e., to obtain a diffraction pattern as a function of momentum transfer for each depth position in the breast.

A classical EDXRD setup consists of a collimated polychromatic X-ray source, a secondary collimation system to select the nominal diffraction angle, and a spectroscopic detector. In our setup, we associate a collimated polychromatic incident pencil beam with convergent secondary collimation targeting (x,y) (Fig. 2 ). This type of system corresponds to a multi-angle EDXRD system, since different diffraction angles will be intercepted (convergent system). Furthermore, intercepting different scattering angles makes it possible to use a larger detector surface for the region to be imaged, hence increasing the systems sensitivity. The use of a thin pencil beam means that the diffraction signal is only emitted from a thin line in the sample, which will be referred to as the Z-axis hereafter.

Another possible solution to inspect the suspicious region would be CSCT $[7,15,26]$. Batchelar et al. [26] used an ADXRD CSCT system working without secondary collimation. This results in an increase of the system sensitivity. Though, the sample to be analyzed must be thin. Otherwise, there would be mixing between different scattering angles and tissues. As we want to analyze the whole breast thickness, this system is not suitable. Ghammraoui et al. [15] compared the performance of two CSCT system configurations: fan-beam and pencil-beam configuration. Fan-beam geometry allows to acquire a whole image slice at once but to avoid mixing of different scattering angles strong collimation is required, which significantly reduces system sensitivity. Pencil beam configuration requires less collimation. Though, measurement lines (or 
translations) and sample rotation are required, which is not always easy in clinical routine. They also investigated the use of multiplexed-beams geometry which is faster than pencil beam configuration but still requires sample rotation typical for CT imaging.

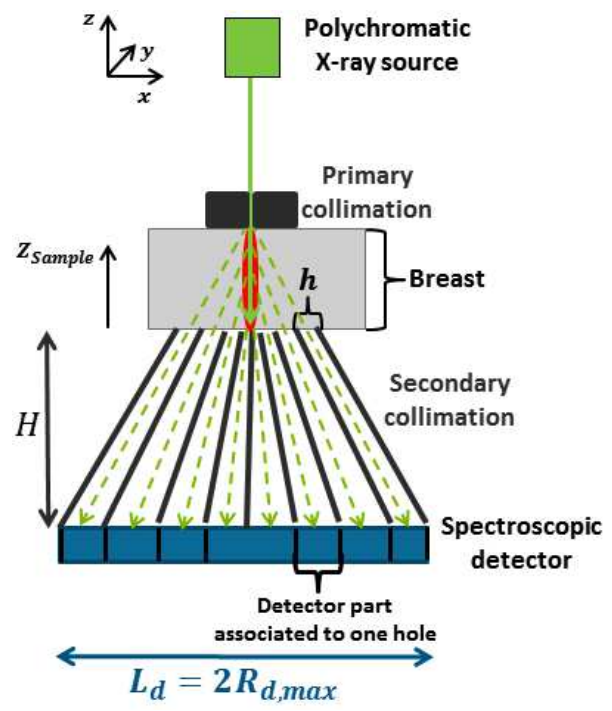

Figure 2: Schematic representation of a system combining EDXRD information at multiple scattering angles: a polychromatic incident pencil beam, a convergent (multifocal) secondary collimation system and a spectroscopic detector.

\subsubsection{Incident $X$-ray beam}

The incident X-ray source was simulated using SpekCalc [27]. The anode material was chosen to be tungsten. The choice of a tungsten anode instead of a molybdenum as normally used in mammography, can be justified by the fact that molybdenum presents low bremsstrahlung intensity compared to its characteristic peaks. In EDXRD, it is important to have a more continuous distribution of photons over the different energies of the incident spectrum in order to cover the required $\chi$-range at the chosen scattering angle. Hence, tungsten is a suitable choice. A $0.2 \mathrm{~mm}$ copper filter was used to suppress photons below $20 \mathrm{keV}$ as these photons would be almost entirely absorbed by photoelectric interaction (Fig. 3) producing a dose deposit without generating any 


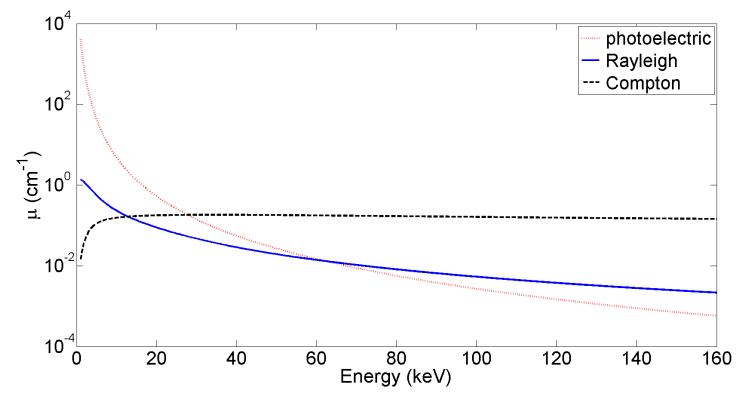

(a) Linear attenuation coefficients.

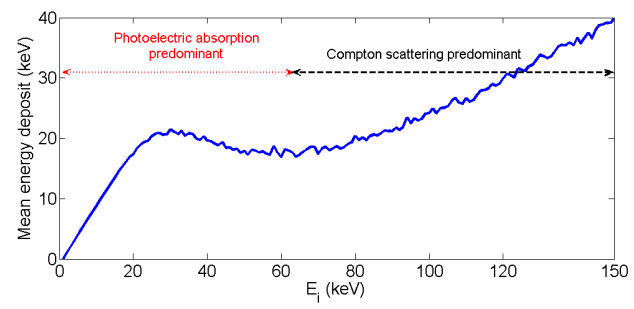

(b) Mean energy deposit.

Figure 3: a) Linear attenuation coefficients of water for different types of interaction. b) Mean energy deposit (multiple interactions included) for one photon in $50 \mathrm{~mm}$ of a material with average chemical composition of breast tissue as a function of incident photon energy (generated with PENELOPE 2008 [28]).

diffraction signal. Similarly, energies above $100 \mathrm{keV}$ appear superfluous as the probability of Rayleigh scattering becomes very low compared to Compton scattering. This is illustrated by Fig. 3 showing the linear attenuation coefficients of water (a) and the mean energy deposit (generated with PENELOPE 2008 [28]) in $50 \mathrm{~mm}$ of a material whose chemical composition is close to average breast tissue (H: $9.82 \%$, C: $33.15 \%$, N: $3.41 \%$, O: $51.96 \%$, P: $0.50 \%$, S: $0.52 \%$, $\mathrm{K}: 0.63 \%$ [29], percentages correspond to mass fractions). Hence, the reference incident X-ray spectrum in this work is between 20 and $100 \mathrm{keV}$. Though, to study the impact of the maximum energy of the incident X-ray spectrum on system performances, a range of tube voltages, between $40 \mathrm{kV}$ and $150 \mathrm{kV}$, was tested. 


\subsubsection{Secondary collimation}

Two possible convergent collimation systems were considered here: a monofocal system and a multifocal system. Monofocal collimation systems only target at one point in the object and require a depth scan, whereas multifocal systems focus on several sample points simultaneously, and can acquire the whole depth in one shot. Both systems require an (x,y)-scan to image the region identified as suspect by mammography. A convergent monofocal system has been considered by Malden and Speller [17] who used a multi-angle approach for baggage inspection. Though, as it is focused on only one voxel this kind of system would also require a scan in the z-direction. This would significantly increase dose delivery and it appears too complex for routine use in practice. Hence, this article only presents results obtained with a multifocal collimation system able to image the whole sample thickness in one go. A schematic representation of a multifocal collimation system is illustrated in Fig. 2.

To obtain the best possible resolution and sensitivity in all directions, a conical secondary collimation setup was chosen, in line with the intrinsic symmetry of XRD. Circular symmetry for the secondary collimation was previously suggested by Harding and Schreiber [30] for instance. However, their system is a mono-angle EDXRD system with one slit focused on only one object voxel, whereas the collimation system proposed here corresponds to a multi-angle EDXRD system with several slits focused on a whole sample line. It is composed of seven hollow cones nested one inside the other. A beam-stop was placed in the center to stop transmission signal. The collimation system in Fig. 2 represents a cross-section of this type of collimation system.

The collimation system was designed without multiplexing, which means that each detector pixel only receives the diffraction signal from a single object voxel. In the case of the presented system, this means that each detector pixel only receives the diffraction signal from a single collimation hole (a concentric annulus in our case) as the system was conceived to adress only one connected depth-region in the target. Therefore, the detector was divided into as many 
parts as the number of holes, and each part was associated with a single hole (Fig. 2). It has to be noted that a detector part contains several pixels. The total detection surface is the same for each part. Fig. 4 illustrates the subdivision of the detector and the definition of a pixel annulus.

Each hole targets a different sample depth position (multifocal collimation) and covers a well defined region in the sample. Collimation height $H$ and hole aperture $h$ were adjusted in order to intercept the required momentum transfer range (combination of incident photon energies and covered scattering angles) between $\chi=1$ and $2.5 \mathrm{~nm}^{-1}$ (Fig. 1) and to obtain a momentum transfer resolution of at least $0.2 \mathrm{~nm}^{-1}$ while maintaining system sensitivity as high as possible. Table 1 summarizes the system parameters used for this study. Resolution was mostly influenced by collimation height and hole size. "Pixel size" (annular thickness) was not limiting if it remained small (around $1 \mathrm{~mm}$ or smaller). The number of holes was fixed in order to have as much holes by respecting a septa thickness of at least $0.3 \mathrm{~mm}$ and by targeting the desired scattering angles. Covered scattering angles range from $0.1^{\circ}$ to $11^{\circ}$, where each hole intercept a different range of angles. The first hole corresponds to low scattering angles (up to $5^{\circ}$ ), whereas the last hole covers the highest scattering angles. Collimator material was simulated as iron, which is less expensive than tungsten and easier to manufacture. Septa thickness was about $1 \mathrm{~mm}$ at detector side and between 0.3 and $0.5 \mathrm{~mm}$ at sample side.

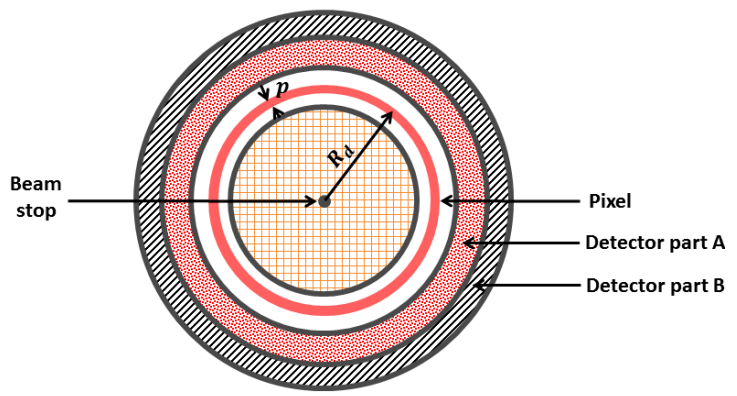

Figure 4: Schematic representation of detector partitioning (detection surface of detector part $\mathrm{A}=$ detection surface of detector part B). One detector part contains more than one pixel. The red pixel, which is represented here, is part of the white detector part. 
Table 1: System parameters. $H$ : collimation height; $h$ : collimation hole size; $p$ : annular thickness; $L_{d}$ : detector dimension.

\begin{tabular}{ccccc}
\hline \hline $\begin{array}{c}\text { Number } \\
\text { of holes }\end{array}$ & $\begin{array}{c}H \\
(\mathrm{~mm})\end{array}$ & $\begin{array}{c}h \\
(\mathrm{~mm})\end{array}$ & $\begin{array}{c}p \\
(\mathrm{~mm})\end{array}$ & $\begin{array}{c}L_{d} \\
(\mathrm{~mm})\end{array}$ \\
\hline 7 & 230 & 0.8 & 1.0 & 100 \\
\hline \hline
\end{tabular}

Obviously, eventhough this kind of collimation allows to collect the scattering signal following the whole annulus associated to one scattering angle, it strongly restricts the number of detected photons compared to the number of incident photons. Coded aperture systems combining EDXRD and ADXRD [31, 32] use less collimation and can also be used to obtain a 2D image. However, this kind of systems seems more adequate for discrete scattering signatures than for continuous tissue signatures. Eventhough the number of detected photons is higher, the amount of information that can be retrieved is not necessarily higher because of ambiguity between scattering angle and momentum transfer (due to multiplexing) with coded apertures, which seems to be more difficult to resolve for amorphous spectra than for crystalline spectra. The amount of information carried by the photons is not the same for each detected photon. We tested to add slight multiplexing to our system in order to increase system sensitivity, but the required dose to detect the small tumor also increased.

\subsubsection{Detector}

For this study, we considered a $5 \mathrm{~mm}$ thick $2 \mathrm{D} 10 \times 10 \mathrm{~mm}^{2}$ spectroscopic CdZnTe detector with $2.5 \mathrm{~mm}$ anode pitch. CdZnTe is a room temperature semiconductor detector with high resistivity $\left(\rho=5 \cdot 10^{10} \Omega \cdot \mathrm{cm}\right)$, good transport properties $\left(\mu \tau=5 \cdot 10^{-3} \mathrm{~cm} \cdot V^{-1}\right)$ and a crystallinity compatible with fabrication of devices measuring several $\mathrm{cm}^{3}$. The thickness of $5 \mathrm{~mm}$ is hence feasible and is well appropriate for the considered range of energies. In a previous study [33], we showed that a spatial resolution of $0.4 \mathrm{~mm}$ can be achieved at $60 \mathrm{keV}$ with this detector geometry using transient signal processing, a technique known as 
subpixel positioning. The advantage of this technique is that it can improve spatial resolution without degrading the spectral response by charge sharing (due to smaller anode size). Here, $1 \mathrm{~mm}$ subpixel size was used for our system simulations. The associated ASIC (Application-Specific Integrated Circuit) was based on IDeFX-HD [34], which combines low noise and low power. In practice, the average energy resolution was about $2.5 \%$ at $122 \mathrm{keV}$.

Using these characteristics, a detector response matrix (DRM), accounting for limited energy resolution of the detector, was obtained using Tasmania, a simulation environment developed in our laboratory. It gives the probability of detecting a given amplitude $A$ knowing the incident photon energy $E_{d}$ on the detector. This detector response model takes into account the following elements: radiation (photons \& photoelectrons) transport based on PENELOPE [28], a 3D electric field model (for applied and weighting fields), 3D charge carrier transport (diffusion, drift, trapping \& Coulomb repulsion) [35], a detailed noise model (detector \& readout electronics) and a behavioral model of analog electronics (shaping, trigger \& peak detector).

\subsubsection{Sensitivity and resolution}

System sensitivity only depends on the geometric parameters of the acquisition system and not on the interaction cross-section of the sample. The sensitivity achieved with detector position $r$ for the position in the sample $Z_{\text {Sample }}$ can be defined as the ratio of the surface (Fig. 5.a), at detector level, of an isotropically radiating point located at $Z_{\text {Sample }}$ to the intercepted detector surface at detector position $r$ :

$$
d S(Z, r)=\int_{0}^{2 \pi} \frac{Z r d r}{4 \pi\left(r^{2}+Z^{2}\right)^{\frac{3}{2}}} d \varphi
$$

where $Z=Z_{\text {Sample }}+H+g$ with $g$ the gap between secondary collimation and sample. Integration over the cylindrical coordinate, $\varphi$, is necessary to consider the whole detector annulus of radius $r$ and thickness $d r$ (Fig. 5.b). To obtain a system sensitivity in the pixel range with a radius, $R_{d}$, from the center to the 


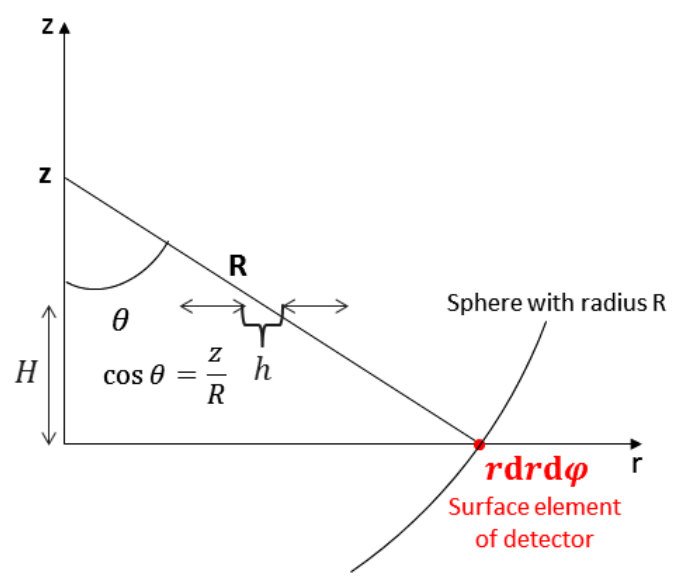

(a) Example for one hole $h$.

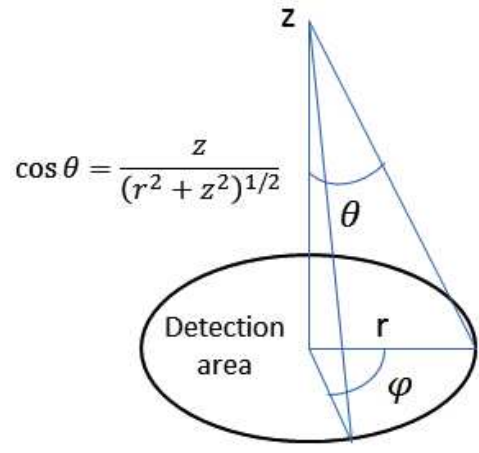

(b) Cylindrical geometry.

Figure 5: Schematic illustration of sensitivity calculations.

sampling point, $Z_{\text {Sample }}$, it is sufficient to integrate all $r$ from which $Z_{\text {Sample }}$ can be "seen" and which are part of the pixel.

Fig. 6.a shows the sensitivity distribution on the detector (detector position $R_{d}$ ) and in the sample (depth position in the sample $Z_{\text {Sample }}$ ) which was calculated analytically. The higher the sensitivity value the more of the photons (isotropically) emitted from $Z_{\text {Sample }}$ can be detected at a given detector position $R_{d}$. A gap of $10 \mathrm{~mm}$ between the secondary collimation and the breast sample was considered to produce maximum sensitivity at the center of the breast. The size of the gap was determined by considering the analytically calculated sensitivity profile in the sample for the case, where the sample and the secondary collimation are in contact. In this case, maximum sensitivity was at $Z_{\text {Sample }}=35 \mathrm{~mm}\left(Z_{\text {Sample }}=0 \mathrm{~mm}\right.$ corresponds to the sample edge at secondary collimation side). The different detector parts were obviously sensitive to different sample zones. Hence, the system is a multifocal collimation system. This multifocal nature was also confirmed by the sensitivity profile in the sample represented in Fig. 6.b, which corresponds to the sum over $R_{d}$ of Fig. 6.a. The systems sensitivity was good almost throughout the samples thickness with its maximum, which is indeed at the center $\left(Z_{\text {Sample }}=25 \mathrm{~mm}\right)$. Because it is 
close to the pencil beam axis (Z-axis) where the diffraction signal originates, the first hole is sensitive to the whole sample thickness (Fig. 6.a), meaning that its spatial resolution is very poor. Global resolution of the system is significantly deteriorated by the first hole, but it allows detection of features in the lower sample regions and thus increases the systems overall sensitivity as a whole.

The overall system performance of the collimation was assessed based on detective quantum efficiency (DQE) and modulation transfer frequency (MTF) calculations $[19,36]$. It has to be noted this is not the commonly used definition of DQE for detectors. Here it indicates the performance of the collimation system associated to a given detector area. This wider concept of DQE was also considered by Starck et al. [37] to characterize gamma camera systems. We adapted these calculations do XRD imaging systems [19]. In photon counting systems (Poisson noise) such as XRD systems, the DQE is the product of the sensitivity and squared modulus of the MTF [36]: DQE $(\nu)=S \cdot \operatorname{MTF}^{2}(\nu)$.

We are able to determine the MTF of one pair hole-pixel $\left(\operatorname{MTF}_{h p}\left(\nu_{z}\right)\right)$. Use of the DQE allows to obtain a synthetical figure of merit combining sensitivity and resolution [19]. The DQE of a pair hole-pixel is given by the squared $\mathrm{MTF}_{h p}\left(\nu_{z}\right)$ weighted by its sensitivity $S_{h p}$. To get the DQE of the whole collimation system, the contributions of the different pairs hole-pixel can simply be summed if there is no multiplexing [36]:

$$
\operatorname{DQE}\left(\nu_{z}\right)=\sum_{\text {pixels }} S_{h p} \cdot \mathrm{MTF}_{h p}^{2}\left(\nu_{z}\right)
$$

The global spatial resolution, $\mathcal{R}$, can be determined from the modulation transfer function curve:

$$
\frac{1}{\mathcal{R}}=2 \int_{0}^{+\infty} \frac{\operatorname{DQE}\left(\nu_{z}\right)}{\operatorname{DQE}(0)} d \nu_{z}=2 \int_{0}^{+\infty} \operatorname{MTF}^{2}\left(\nu_{z}\right) d \nu_{z}
$$

This definition is consistent with the full width at half maximum (FWHM) for a rectangular gate-shaped point spread function. Angular $\mathrm{MTF}_{h p}\left(\nu_{\theta}\right)$ is determined by geometrical considerations linking $z$ and $\theta$, and momentum transfer $\mathrm{MTF}_{h p}\left(\nu_{\chi}\right)$ can be calculated using the relationship between $\theta$ and $\chi$ (Eq. 1). 
The calculated overall system resolutions are summarized in table 2. With $9.6 \mathrm{~mm}$ in the direction of the samples thickness, the spatial resolution is low, but it is nevertheless better than other XRD systems, which do not offer any depth resolution. Some XRD imaging techniques such as CSCT [7, 16] and coded aperture coherent spectral imaging $[31,38]$ allow to obtain better spatial resolution in beam direction. Greenberg et al. [38], for instance, obtained a spatial resolution of $5 \mathrm{~mm}$ in beam direction. However, contrast is lost due to multiplexing inherent in coded aperture methods. In CSCT the resolution is good but in clinical routine it is not always easy to rotate while imaging, especially in breast imaging. We seek to propose an imaging system able to acquire the whole sample thickness in one go with a satisfying spatial resolution and having a good sensitivity while maintaining the best possible momentum transfer resolution without the need of rotating movements.

The momentum transfer resolution meets the constraint of $0.2 \mathrm{~nm}^{-1}$ for almost every energy tested due to adequate angular resolution. Integration over the whole incident X-ray spectrum (between 20 and $100 \mathrm{keV}$ ) leads to an average momentum transfer resolution of about $0.07 \mathrm{~nm}^{-1}$. However, the calculations did not take the detectors energy resolution into account because we were interested in proposing the best possible collimation system. Doing so slightly deteriorates the momentum transfer resolution, as this resolution depends on both angular and spectral resolution:

$$
\Delta \chi=\frac{E}{2 h c} \cdot \cos \left(\frac{\theta}{2}\right) \Delta \theta+\frac{1}{h c} \sin \left(\frac{\theta}{2}\right) \Delta E
$$

If a mean energy resolution of $3 \mathrm{keV}$ is considered, this corresponds to $5 \%$ at $60 \mathrm{keV}$. This leads to a resolution of $0.1 \mathrm{~nm}^{-1}$ at $2 \mathrm{~nm}^{-1}$. At $20 \mathrm{keV}$ this resolution will be lower and $100 \mathrm{keV}$ it will be higher, but in average the global momentum transfer resolution is not worse than the required $0.2 \mathrm{~nm}^{-1}$. In the following simulation study both contributions (angular resolution of the collimation and energy resolution of the detector) are taken into account. 


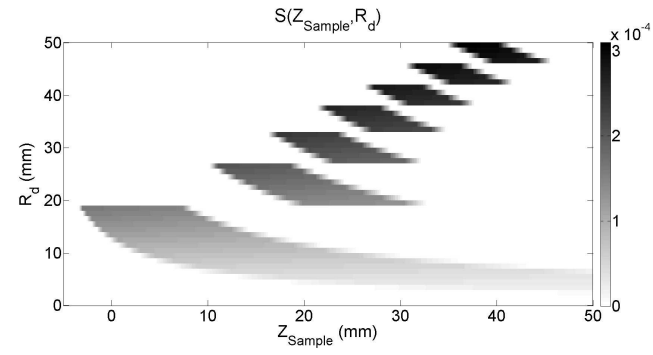

(a) Sensitivity distribution.

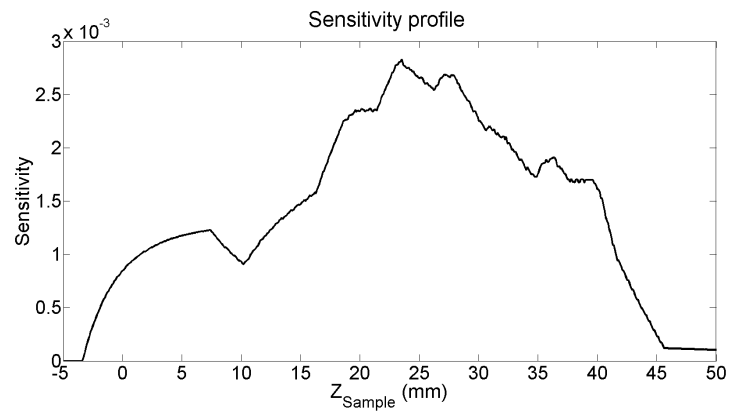

(b) Sensitivity profile.

Figure 6: Sensitivity distribution and sensitivity profile of the simulated X-ray diffraction system across the sample (sample starts at 0 and ends at $50 \mathrm{~mm}$ ).

Table 2: Resolutions of the X-ray diffraction system considered (the spectral resolution of the detector not taken into account).

\begin{tabular}{cccccc}
\hline \hline $\begin{array}{c}\text { Spatial } \\
(\mathrm{mm})\end{array}$ & $\begin{array}{c}\text { Angular } \\
\left({ }^{\circ}\right)\end{array}$ & $\begin{array}{c}\chi \text { at } 20 \mathrm{keV} \\
\left(\mathrm{nm}^{-1}\right)\end{array}$ & $\begin{array}{c}\chi \text { at } 60 \mathrm{keV} \\
\left(\mathrm{nm}^{-1}\right)\end{array}$ & $\begin{array}{c}\chi \text { at } 100 \mathrm{keV} \\
\left(\mathrm{nm}^{-1}\right)\end{array}$ & $\begin{array}{c}\chi \text { at } 150 \mathrm{keV} \\
\left(\mathrm{nm}^{-1}\right)\end{array}$ \\
\hline 9.6 & 0.21 & 0.029 & 0.09 & 0.15 & 0.22 \\
\hline \hline
\end{tabular}




\subsection{Simulations}

Our X-ray diffraction system's capacity to distinguish between carcinoma and fibroglandular tissue was assessed using Monte-Carlo simulations of the whole system and a breast phantom. These simulations were performed with PENELOPE [28], by adapting cross-sections for coherent scattering to take intra- and intermolecular radiation interference, i.e., X-ray diffraction, into account. Cross-sections used in PENELOPE for Rayleigh scattering only contain the Thomson cross-section and the coherent scatter form factor (Eq. 2). We replaced these cross-sections by the diffraction signatures determined by [10] (Fig. 1.b).

Simulations take into account all kinds of interaction (Rayleigh scattering, Compton scattering, Photoelectric absorption, multiple scattering, fluorescence) and all possible locations of interaction (collimation, phantom, detector).

\subsubsection{Breast phantom}

The simulated breast phantom was a $50 \mathrm{~mm}$ thick, and $150 \mathrm{~mm}$ diameter cylinder of adipose tissue containing an ellipsoid of fibroglandular tissue (axis in $\mathrm{x}$ - and $\mathrm{y}$-direction: $70 \mathrm{~mm}$ ) corresponding to a region with altered composition. To assess the impact of the thickness (i.e. the axis in z-direction) of fibroglandular tissue, this ellipsoid was simulated in a range of thicknesses: 20, 30 and $40 \mathrm{~mm}$. This allowed us to verify whether high density breast tissue can be imaged with XRD. A small spherical carcinoma nodule was located in the center of the phantom, in the case of a phantom with tumor. The diameter of this nodule was about $4 \mathrm{~mm}$, which corresponds to a very small tumor size. In scintimammography, for instance, tumors smaller than $6 \mathrm{~mm}$ are difficult to detect $[39,40]$. Fig. 7 shows a schematic representation of the breast phantom.

As system sensitivity is not the same over the whole of the samples thickness, it is important to study the impact of tumor position on its detectability. The tumor was always located in the fibroglandular mass at this location will result in doubtful mammography results. Therefore, tumor position variation study was realized with $40 \mathrm{~mm}$ fibroglandular thickness. Four tumor offset positions 
were tested: $+20 \mathrm{~mm},+10 \mathrm{~mm},-10 \mathrm{~mm}$ and $-20 \mathrm{~mm}$. A positive tumor offset corresponds to a greater distance between tumor and detector, while a negative offset corresponds to a shorter distance.

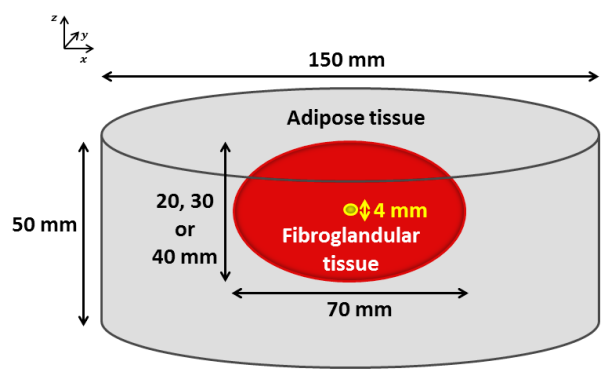

Figure 7: Simulated breast phantom presenting a nodule (yellow sphere) composed of pure carcinoma. are equivalent to scans in x-direction.) were simulated with and without tumor for a fibroglandular thickness of $30 \mathrm{~mm}$ and incident spectra at $60 \mathrm{kVp}$ and $100 \mathrm{kVp}$. The scan step was chosen to be $1 \mathrm{~mm}$.

\subsection{Data analysis}

\subsubsection{Separation power and required incident photon number}

The contrast to noise ratio $(\mathrm{CNR})$ is a measure of the separation power of a given imaging system and is defined as:

$$
C N R=\frac{\Delta M}{\sigma}
$$

where $\Delta M=M_{A}-M_{B}$ corresponds to the difference between the expected signal intensities $M_{A}$ and $M_{B}$ produced by materials $\mathrm{A}$ and $\mathrm{B}$, and $\sigma$ corresponds to the standard deviation of the noise.

Diffraction measurements follow a Poisson law, but for CNR calculations a Gaussian approximation $\left(\mu=\sigma^{2}=\lambda\right.$, where $\lambda$ is the Poisson parameter $)$ of photon noise was used.

If $\mathrm{A}$ and $\mathrm{B}$ are the two tissue types to be distinguished (carcinoma and fibrog- 
landular tissue), using the Gaussian approximation, the squared CNR is given by:

$$
C N R^{2}=\sum_{i} \frac{\left(M_{A, i}-M_{B, i}\right)^{2}}{\sigma_{i}^{2}}
$$

where $M_{A}$ and $M_{B}$ are the expected diffraction signatures of $\mathrm{A}$ and $\mathrm{B}$ normalized by the number of incident photons, and $\sigma_{i}^{2}=M_{A, i}+M_{B, i}$ the standard deviation of $M_{A}-M_{B}$ on channel $i$.

In fact, the $\mathrm{CNR}^{2}$ defined above corresponds to the quadratic distance between the two distributions, A and B, per incident photon. Hence, the inverse of the $\mathrm{CNR}^{2}$ corresponds to the number of photons, $N_{p h, 1 \sigma}$, requested to obtain a distance of one standard deviation between the two distributions. This is a very low-level separation. Hereafter, a separation of $3 \sigma$ will be considered:

$$
N_{p h, 3 \sigma}=\frac{9}{C N R^{2}}
$$

\subsubsection{Dose estimation}

The radiation dose was estimated using the incident X-ray spectrum, $S_{i n c}$, the mean energy deposit for one photon in $50 \mathrm{~mm}$ of breast tissue similar material, $E_{\text {mean }}$, as a function of incident photon energy, $E_{i}$ (Fig. 3.b), and the calculated incident photon number, $N_{p h}$, required to obtain the desired separation between XRD spectra with and without carcinoma.

The mean total energy deposit per photon, $D_{E}$, for a given incident spectrum is calculated using:

$$
D_{E}=\frac{\sum_{E_{i}} E_{\text {mean }}\left(E_{i}\right) \cdot S_{\text {inc }}\left(E_{i}\right)}{\sum_{E_{i}} S_{\text {inc }}\left(E_{i}\right)}
$$

The result is expressed in $\mathrm{keV}$. For an incident spectrum with a maximum energy of $100 \mathrm{keV}$, the mean total energy deposit will be about $18.88 \mathrm{keV}$. Knowing the required number of incident photons, the required mean dose is given by: 


$$
D=\frac{D_{E} \cdot 1.6 \cdot 10^{-16} \cdot N_{p h}}{m_{i r r}}
$$

where $1.6 \cdot 10^{-16}$ is the conversion factor to transform $\mathrm{keV}$ into joules $(\mathrm{J})$, and $m_{i r r}$ corresponds to the directly irradiated mass. In the present case, the irradiated mass is about $5 \cdot 10^{-8} \mathrm{~kg}$ (i.e. pencil beam surface $\times$ sample thickness $\times$ sample density $\left.=10^{-6} \mathrm{~m}^{2} \times 50 \cdot 10^{-3} \mathrm{~m} \times 1 \mathrm{~kg} \cdot \mathrm{m}^{-3}\right)$.

Note that this dose corresponds indeed to the total dose deposited in the whole breast thickness. The mean energy deposit for one photon $E_{\text {mean }}$ was obtained by Monte-Carlo simulations taking into account every possible interaction within the whole sample thickness.

\subsubsection{Reconstruction method}

The simulated scan measure $m$ is modeled linearly by:

$$
m\left(A, R_{d}, x\right)=\sum_{Z, i} \operatorname{Resp}\left(A, R_{d}, Z, i\right) \cdot t(Z, i, x)
$$

where $x$ corresponds to the scan position and $\operatorname{Resp}\left(A, R_{d}, Z, i\right)$ to the system response matrix depending on the detected amplitude $A$, the position on the detector $R_{d}, Z$ and the tissue type $i$ (adipose tissue, fibroglandular tissue, carcinoma). $t(Z, i, x)$ is a coefficient indicating whether tissue $i$ is present at the position $Z$ and detected at scan position $x$. As there is no tissue mixing in the breast phantoms considered here, it is either equal to zero (tissue $i$ not present) or to one (tissue $i$ present).

The aim of reconstruction is to process measurements, $m\left(A, R_{d}, x\right)$, to estimate the material coefficients, $t(Z, i, x)$, which can be represented in a color image, using material $i$ as a single color channel.

Methods using material decomposition were also suggested by other authors, Westmore et al. [7] who proposed a non-negative least squares fitting algorithm to estimate the amount of a given material, or Ghammraoui et al. [16] who suggested a version of maximum likelihood expectation maximization algorithm with ordered subsets. 
In this study, we used the maximum likelihood expectation maximization algorithm for reconstruction[41]. This algorithm is based on the iterative maximization of the log-likelihood function, which is very common in the likelihood maximization framework.

The estimated measurement, $\tilde{m}$, can be written assuming an ideal direct system operator, $\mathcal{F}$ :

$$
\tilde{m}\left(A, R_{d}, x\right)=\sum_{Z, i} \mathcal{F}\left(A, R_{d}, Z, i\right) \cdot t(Z, i, x)
$$

The most significant noise source in diffraction is photon noise, which by its nature follows a Poisson law with parameter $\sum_{Z, i} \mathcal{F}\left(A, R_{d}, Z, i\right) \cdot t(Z, i, x)$. A maximum likelihood approach consists in determining:

$$
\hat{t}=\underset{t \geq 0}{\arg \max } P(m \mid t)
$$

An iterative solution is thus given by the following algorithm [41]:

$\hat{t}^{n+1}(Z, i, x)=\frac{\hat{t}^{n}(Z, i, x)}{\sum_{A, R_{d}} \mathcal{F}\left(A, R_{d}, Z, i\right)} \sum_{A, R_{d}} \frac{\mathcal{F}\left(A, R_{d}, Z, i\right) \cdot m\left(A, R_{d}, x\right)}{\sum_{Z, i} \mathcal{F}\left(A, R_{d}, Z, i\right) \cdot \hat{t}^{n}(Z, i, x)}$

where $\hat{t}^{n+1}$ is the estimate of $t$ at the nth iteration. Choosing a positive value for $\hat{t}^{0}$ will ensure that the final results are non-negative. Here, a uniform initialization was used.

\section{Results and discussion}

Fig. 8 shows two sample XRD spectra (normalized by the number of incident photons) to be distinguished; the fibroglandular region was $30 \mathrm{~mm}$ thick and the incident spectrum had a maximum energy of $100 \mathrm{keV}$. These spectra were simulated for $\mathrm{x}=0$ and $\mathrm{y}=0$ corresponding to the phantom center in $\mathrm{x}, \mathrm{y}-$ plane. All the following results are given for this position except the scan slice reconstruction, where x-position varied. The gap between secondary collimation and object was $10 \mathrm{~mm}$ for each simulation in this work. 
The segments visible in the spectra and even more clearly in their difference correspond to the limits of the different collimation holes. The dotted lines in Fig. 8.c indicate these limits. By summing the normalized spectra from Fig. 8 in both directions, the object depending global sensitivity can be determined, which takes into account geometrical factors as well as the sample interaction cross-section. It corresponds to the ratio between the number of incident photons on the sample and the number of detected photons. This is not exactly the same quantity as defined in section 3.1.4, which is purely geometrical (which proportion of photons emitted isotropically at a given $Z$ can be detected by the system) and does not take into account interaction cross-section in the sample.

In this case, object depending global sensitivity is approximately $2 \cdot 10^{-3}$. Multiplication of this value by the number of incident photons gives the total number of photons detected. The $C N R^{2}$ per incident photon is about $2 \cdot 10^{-6}$, which is very low, explaining why the differences between the two XRD spectra (without tumor (Fig. 8.a); with tumor (Fig. 8.b)) are hardly visible. Fig. 8.c shows the difference spectrum in absolute value. The difference to be detected is only in the order of a few percent (difference values per pixel and per channel between $10^{-8}$ and $10^{-7}$ compared to XRD spectrum values per pixel and per channel of about $\left.10^{-6}\right)$.

Hereafter, the photon number and associated dose are given for a separation of $3 \sigma$ between XRD spectra with and without tumor. This degree of separation corresponds to an almost perfect separation based on the associated analytically calculated ROC curve [42]. The number of incident photons required is determined using equation 9 and will be around $5 \cdot 10^{6}$. The associated dose is calculated using equation 11 . Thus, all given dose values correspond to the dose delivered to the irradiated sample volume over the whole sample thickness.

\subsection{Fibroglandular thickness}

The results for the required photon number and the corresponding dose for an incident spectrum with a maximum energy of $100 \mathrm{keV}$ and different fibroglandular thicknesses are summarized in table 3. Fibroglandular thickness 


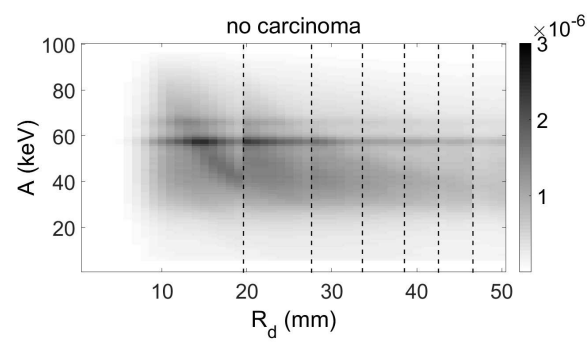

(a) XRD spectrum without tumor.

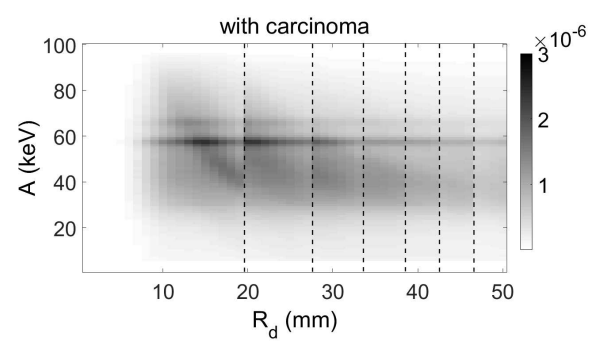

(b) XRD spectrum with tumor.

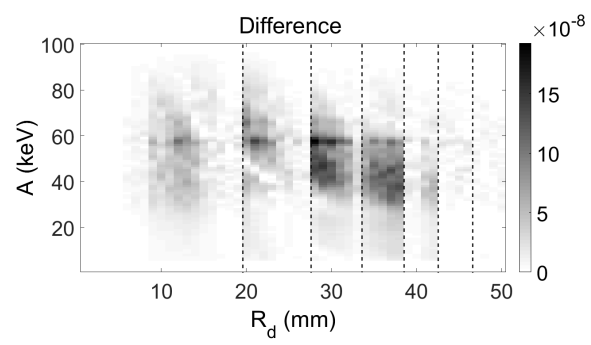

(c) Difference spectrum (absolute value).

Figure 8: Simulated XRD spectra $(\mathrm{x}=0, \mathrm{y}=0,10 \mathrm{~mm}$ gap between sample and secondary collimation) normalized for the number of incident photons and the absolute value of their difference. Fibroglandular thickness: $30 \mathrm{~mm}$; incident spectrum maximum energy: $100 \mathrm{keV}$. The difference to be detected is minute compared to XRD spectrum values. Dotted lines correspond to the limits of the detector parts addressed by the different holes. 
Table 3: Incident photon number and dose required to obtain a separation of $3 \sigma$ for an incident spectrum with maximum energy of $100 \mathrm{keV}$.

\begin{tabular}{ccc}
\hline \hline $\begin{array}{c}\text { Fibroglandular } \\
\text { thickness }(\mathrm{mm})\end{array}$ & $\begin{array}{c}\text { Required photon } \\
\text { number }\end{array}$ & $\begin{array}{c}\text { Required dose } \\
(\mathrm{mGy})\end{array}$ \\
\hline 20 & $3.85 \cdot 10^{6}$ & 0.232 \\
30 & $4.20 \cdot 10^{6}$ & 0.253 \\
40 & $4.12 \cdot 10^{6}$ & 0.244 \\
\hline
\end{tabular}

has a negligible impact on the number of photons required, thus averaging at about $4 \cdot 10^{6}$ for a corresponding dose of about $0.24 \mathrm{mGy}$. This dose is low compared to conventional mammography, where the dose delivered is between 1 and 3 mGy [43-45].

Hence, scattering signatures for cancerous tissues and fibroglandular tissues are sufficiently decorrelated to be distinguished even when the number of detected photons is small. Furthermore, as the required dose does not significantly vary with fibroglandular tissue thickness, high density breast regions pose no problems in XRD, unlike in conventional mammography. In fact, as used tube voltage is significantly higher than in classical mammography photons have higher energy and reduction of expected counts due to increased breast density is less significant. Moreover, the incident spectrum was filtered in order to remove photons at energies below $20 \mathrm{keV}$ in order to avoid unnecessary dose deposit by photons that are almost completely absorbed, whereas mammography typically uses photons at these energies.

\subsection{Influence of different maximum energies}

Fig. 9 shows the dose received by samples analyzed using our optimized system, as a function of the maximum energy of the incident spectrum for a phantom with $40 \mathrm{~mm}$ thick fibroglandular tissue section with a tumor at the center of the field of view. A minimum was observed at 60 and $70 \mathrm{keV}$ corresponding to a 
dose of about 0.21 mGy. For incident spectra with lower maximum energy, the combination of photon energy and intercepted angles is less appropriate and absorption remains higher than coherent scattering. At higher energies, the probability of absorption and coherent scattering continue to decrease, whereas Compton scattering probability remains constant. Hence, the proportion of signal due to Compton scattering increases steadily with increasing photon energy, causing blurring of the measured XRD spectrum and unnecessary radiation exposure. However, even though the received dose increases with higher maximum energy of the incident spectrum, the total dose still remains low and acceptable compared to conventional mammography.

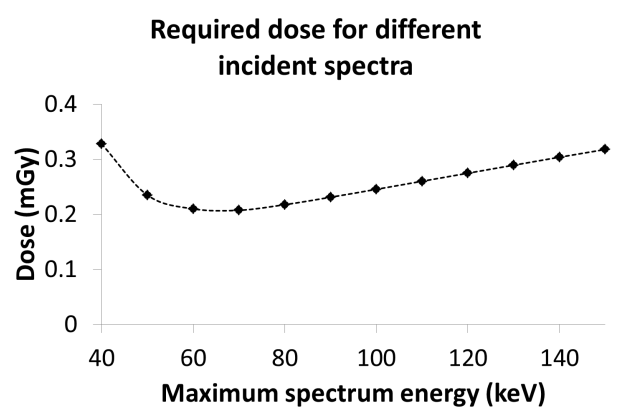

Figure 9: Dose required to separate ( $3 \sigma$ separation) phantoms (40 $\mathrm{mm}$ fibroglandular thickness) with and without tumor as a function of the maximum energy of the incident X-ray spectrum.

\subsection{Impact of tumor position}

The dose required to distinguish between spectra with and without tumor for different tumor offsets and maximum incident spectrum energies are presented in Fig. 10. If the tumor is located in the center (no offset), the required dose is the lowest. In fact, the maximum of sensitivity is in the center and the tumor can be "seen" by five holes (Fig. 6.a). If a tumor offset is introduced, the difference between spectra with and without tumor will not be detected by the same holes, as the system has a variable sensitivity distribution. The first hole always detects part of the difference, as it is sensitive throughout the sample 
thickness. Required doses are slightly higher for other tumor positions but as long as the tumor is in the field of view (sensitivity zone), these doses stay very small. The detection of the tumor for the $+20 \mathrm{~mm}$ offset, where the tumor was positioned at $Z_{\text {Sample }}=45 \mathrm{~mm}$, requires an approximately 5 -fold higher dose. This position corresponds to a very low sensitivity region for the presented system configuration. However, the total dose received still remains not higher than with conventional mammography (1-3 mGy).

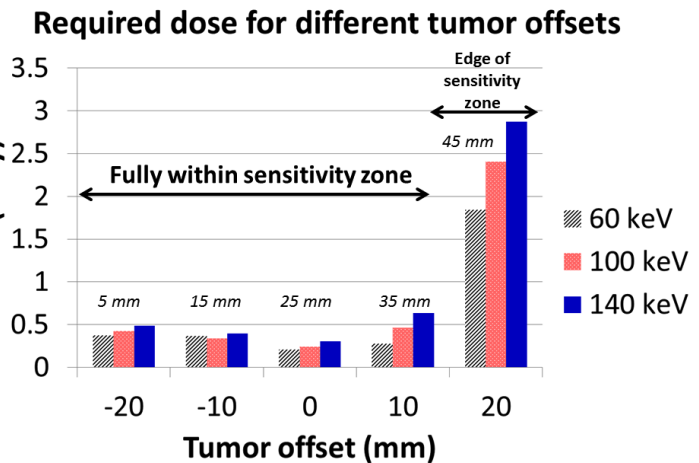

Figure 10: Dose required to distinguish ( $3 \sigma$ separation) between spectra with and without carcinoma depending on the position of the tumor within the sample (depth).

\subsection{Reconstruction results}

Fig. 11 shows an example of a simulated XRD spectrum for a phantom (30 mm fibroglandular tissue thickness, $100 \mathrm{kVp}$ incident spectrum) with a tumor at its center, for scan position $\mathrm{x}=0$, corresponding to the center of the phantom in the $\mathrm{x}, \mathrm{y}$-plane. The number of incident photons was about $5 \cdot 10^{6}$, corresponding to approximately $10^{4}$ detected photons and to a dose deposit of about $0.3 \mathrm{mGy}$. This dose is consistent with the required dose determined in the previous parts of the study and with the dose given by Ghammraoui et al. [15]. With an incident dose of about 0.5 mGy a small tumor of similar size could be reconstructed in a CSCT image when pencil beam geometry was used.

The number of photons per channel is very low, producing a relatively noisy spectrum. However, the number of incident photons is within the order of 
magnitude determined previously to obtain a $3 \sigma$ separation between phantoms with and without tumor.

Each scan slice was simulated using the same incident photon parameters, and the image of the breast phantom was reconstructed (Fig. 12) using the previously presented reconstruction method. It should be noted that the field of view for the image was set smaller than the phantom height. Hence, only $5 \mathrm{~mm}$ of adipose tissue is visible on the upper and lower parts of the image, rather than $10 \mathrm{~mm}$.

This reconstruction clearly shows that the tumor will only be reconstructed if it is actually in the simulated phantom slice (Fig. 12.b and fig 12.d), and therefore no false positive results should be produced for a tumor positioned in the center of the phantom. The tumor is sharper and more intense in the reconstruction at $60 \mathrm{kVp}$. This is consistent with the fact that the optimal incident spectrum to detect the small tumor was found to be at $60 \mathrm{kVp}$. Hence, using this incident spectrum the best tumor reconstruction should be obtained. Though, compared to reconstructions at $60 \mathrm{kVp}$, reconstructions at $100 \mathrm{kVp}$ present fewer edge artifacts at the limit between fibroglandular tissue and adipose tissue, especially at low depths within the sample. This difference is due to lack of information in the momentum transfer space at low depths. In fact, as can be seen in Fig. 6.a, the first hole in the collimation system is the only hole sensitive to low depth values. Though the corresponding scattering angles are also low because the corresponding detector part is close to the center in the x,y-plane (small $R_{d}$, Fig. 2 and Fig. 4). Thus, to cover the same momentum transfer range as for other holes intercepting higher scattering angles (higher $R_{d}$ values), higher energies are needed. It appears that the information obtained at $60 \mathrm{keV}$ maximum energy is not sufficient to distinguish between fibroglandular and adipose tissue. Reconstruction artifacts (i.e. detection of carcinoma) in the upper corners of the images at $60 \mathrm{kVp}$ are also caused by the lack of information in momentum transfer space at this energy level. As for low depths, these high depth values are only covered by the first hole with very low diffraction angles. In addition to this, these edges correspond to very low system sensitivity 
(Fig. 6.b), which further decreases the amount of available information in these regions. Tumor detection in these regions would not be reliable for the given position of the system especially at $60 \mathrm{keV}$.

To conclude, the detection of a small tumor situated in regions covered also by another hole than the first one is optimum (in terms of dose received) with a $60 \mathrm{kVp}$ incident spectrum, for the considered system. This detection corresponds to a binary task, presence or absence of a tumor, where it is not necessary to identify the different tissue types present in each sample voxel. Reconstruction of an image slice is more complex task. Here, we require spatial information concerning each tissue type everywhere in the sample. In this case, energies higher than $60 \mathrm{keV}$ provide useful information for regions imaged with low scattering angles. If the collimation system was conceived in a way that edge regions were also covered by other holes, i.e. higher scattering angles, edge artifacts might be avoided even with an incident spectrum at $60 \mathrm{kVp}$.

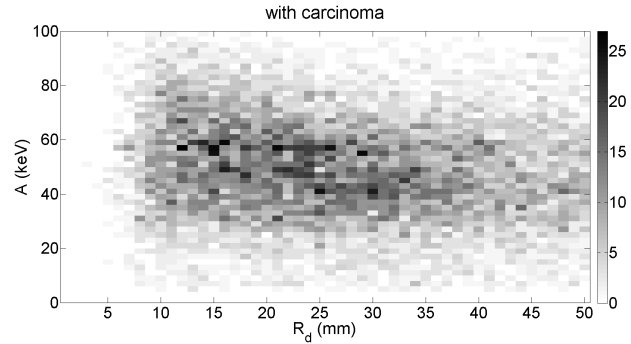

Figure 11: Example of a simulated XRD spectrum with a tumor at its center for scan position $\mathrm{x}=0$ (center in $\mathrm{x}, \mathrm{y}$-plane): $30 \mathrm{~mm}$ thick fibroglandular region, $100 \mathrm{kVp}$ incident spectrum, $5 \cdot 10^{6}$ incident photons.

\section{Conclusion}

The present study indicates that coherent scattering of X-rays appears as a very promising technique to classify breast tissues when classical mammography gives an unclear result, and to reduce the number of unnecessary breast biopsies.

We dimensioned an XRD system, in particular the secondary collimation (hole size, collimation height, number of holes, sensitivity distribution), com- 


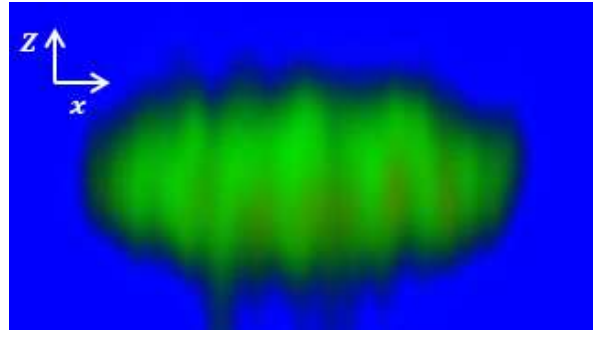

(a) No tumor $(100 \mathrm{kVp})$.

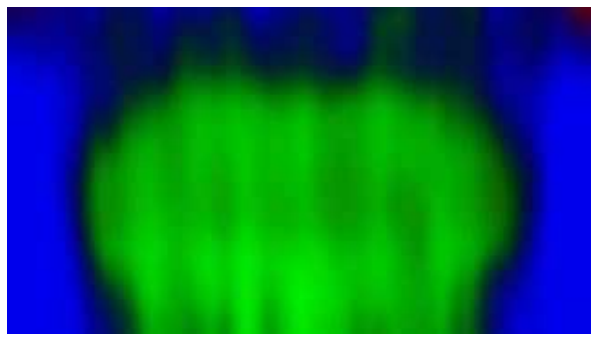

(c) No tumor $(60 \mathrm{kVp})$.

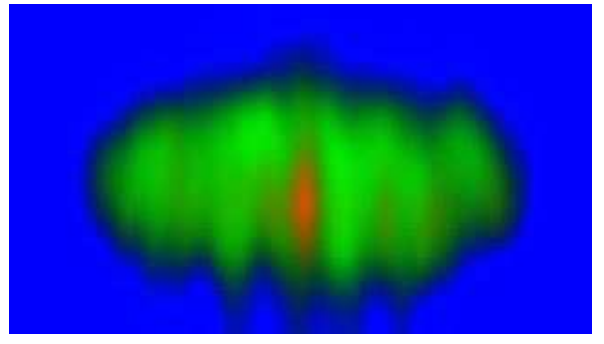

(b) With tumor $(100 \mathrm{kVp})$.

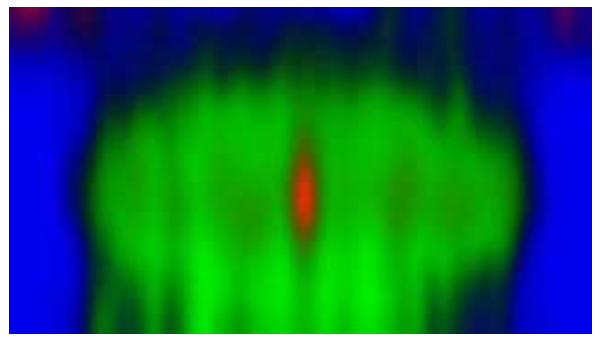

(d) With tumor $(60 \mathrm{kVp})$.

Figure 12: Reconstructed images for incident spectra with $100 \mathrm{keV}$ or $60 \mathrm{keV}$ maximum energy (blue $=$ adipose tissue, green $=$ fibroglandular tissue, red $=$ carcinoma, black $=$ blue + green $)$. 
bining EDXRD information at different scattering angles, for use as a second control after conventional mammography. In this article, we evaluated its performance in breast imaging in terms of separation power. Therefore, XRD spectra for phantoms with and without tumor were used and the required dose to distinguish between these two kinds of spectra was calculated.

It was found that the sensitivity and specificity of the method were good even when the dose delivered was moderate. To achieve a $3 \sigma$ separation, a dose around $0.3 \mathrm{mGy}$ is needed if the tumor is situated in the center of the breast. This dose is very acceptable compared to conventional mammography (1-3 mGy). When the tumor was not in the center of the breast, the required dose increased slightly, but remained very low provided the tumor was located within the field of sensitivity.

Delivered dose is also impacted by the choice of the incident X-ray spectrum used for imaging. In fact, the number of photons, i.e. the incident dose, necessary to detect the tumor depends on the amount of information carried by each detected photon after coherent scattering. This information corresponds to its momentum transfer value, given by the combination of photon energy and its scattering angle. The detected scattering angles are determined by the secondary collimation. Testing of incident tungsten spectra at different tube voltages showed that for the present system the combination of scattering angles and photon energies was the best with an incident spectrum at $60 \mathrm{kVp}$. A system with a different distribution of intercepted scattering angles will have an optimal incident spectrum at a different tube voltage.

As the detection of tumors in dense breasts are often a problem in classical mammography, the impact of varying breast density on separation power of the system was tested. It turned out that different breast densities do not affect the detectability of the tumor or the order of magnitude of the required dose. Hence, XRD imaging with the presented system appears to be a well adapted solution at a second control level after conventional mammography. Though, mammography does only allow to identify a suspicious region in the plane and not the exact location of the possible tumor. Therefore, an x,y-scan of this 
region is required, but scan-time appears to be acceptable. With an incident flux of about $1.5 \cdot 10^{8}$ photons per $\mathrm{cm}^{2}$ per $\mathrm{mAs}$ at $1 \mathrm{~m}$ distance (tube current of about $10 \mathrm{~mA}$ ), scan-time for $10 \mathrm{~cm}^{3}$ would be about 2 seconds. A scan slice in $\mathrm{x}$-direction was simulated to assess the imaging qualities of the proposed XRD system. Reconstruction results confirmed that the small tumor can be detected using this system, although spatial resolution was poor compared to other imaging techniques.

In future work, it will be necessary to manufacture the XRD system presented here so as to be able to make experimental measurements in order to confirm the simulation results. The impact of variability of breast thickness and tissue scattering signatures should also be studied.

\section{References}

[1] P. C. Johns, M. J. Yaffe, X-ray characterisation of normal and neoplastic breast tissues, Physics in Medicine and Biology 32 (1987) 675-695.

[2] S. H. Evans, D. A. Bradley, D. R. Dance, J. E. Bateman, C. H. Jones, Measurement of small-angle photon scattering for some breast tissues and tissue substitute materials, Physics in Medicine and Biology 36 (1991) 7-18.

[3] S. Pani, G. Royle, R. Speller, A. Castoldi, A. Galimberti, C. Guazzoni, Use of a novel controlled drift detector for diffraction enhanced breast imaging, Nuclear Instruments and Methods in Physics Research Section A: Accelerators, Spectrometers, Detectors and Associated Equipment 573 (2007) $133-136$.

[4] G. J. R. A. Taibi, A Monte Carlo simulation study to investigate the potential of diffraction enhanced breast imaging, Nuclear Science, IEEE Transactions on 3 (2000) $1581-1586$.

[5] D. Cunha, A. Tomal, M. Poletti, Diffraction enhanced breast imaging through Monte Carlo simulations, Nuclear Instruments and Methods in 
Physics Research Section A: Accelerators, Spectrometers, Detectors and Associated Equipment 652 (2011) 878-882.

[6] G. Harding, M. Newton, J. Kosanetzky, Energy-dispersive X-ray diffraction tomography, Physics in Medicine and Biology 35 (1990) 33-41.

[7] M. Westmore, A. Fenster, I. Cunningham, Tomographic imaging of the angular-dependent coherent-scatter cross section, Medical Physics 24 (1997) 3-10.

[8] J. Griffiths, G. Royle, J. Horrocks, A. Hanby, S. Pani, R. Speller, Angular dispersive diffraction microCT of small breast tissue samples, Radiation Physics and Chemistry 77 (2008) 373-380.

[9] S. Pani, E. Cook, J. Horrocks, J. Jones, R. Speller, Characterization of breast tissue using energy-dispersive X-ray diffraction computed tomography, Applied Radiation and Isotopes 68 (2010) 1980-1987.

[10] G. Kidane, R. D. Speller, G. J. Royle, A. M. Hanby, X-ray scatter signatures for normal and neoplastic breast tissues, Physics in Medicine and Biology 44 (1999) 1791-1802.

[11] M. J. Farquharson, K. Geraki, The use of combined trace element XRF and EDXRD data as a histopathology tool using a multivariate analysis approach in characterizing breast tissue, X-Ray Spectrometry 33 (2004) 240-245.

[12] R. J. LeClair, M. M. Boileau, Y. Wang, A semianalytic model to extract differential linear scattering coefficients of breast tissue from energy dispersive x-ray diffraction measurements, Medical Physics 33 (2006) 959-967.

[13] A. Chaparian, M. Oghabian, V. Changizi, M. Farquharson, The optimization of an energy-dispersive X-ray diffraction system for potential clinical application, Applied Radiation and Isotopes 68 (2010) 2237-2245. 
[14] M. A. O. Ali Chaparian, Introducing an Optimized Method for Obtaining X-ray Diffraction Patterns of Biological Tissues, Iranian Journal of Medical Sciences 8 (2012) 9-17.

[15] B. Ghammraoui, L. M. Popescu, A. Badal, Monte carlo evaluation of the relationship between absorbed dose and contrast-to-noise ratio in coherent scatter breast CT, in: Proc. SPIE 9412, Medical Imaging 2015, 2015.

[16] B. Ghammraoui, A. Badal, L. M. Popescu, Maximum-likelihood estimation of scatter components algorithm for x-ray coherent scatter computed tomography of the breast, Physics in Medicine and Biology 61 (2016) 31643179 .

[17] C. Malden, R. Speller, Cdznte array for the detection of explosives in baggage by energy-dispersive X-ray diffraction signatures at multiple scatter angles, Nuclear Instruments and Methods in Physics Research, Section A: Accelerators, Spectrometers, Detectors and Associated Equipment 449 (2000) 408-415.

[18] D. O'Flynn, C. B. Reid, C. Christodoulou, M. D. Wilson, M. C. Veale, P. Seller, D. Hills, H. Desai, B. Wong, R. Speller, Explosive detection using pixellated X-ray diffraction (PixD), Journal of Instrumentation 8 (2013) P03007.

[19] F. Marticke, C. Paulus, G. Montémont, O. Michel, J. I. Mars, L. Verger, Multi-angle reconstruction of energy dispersive X-ray diffraction spectra., in: Workshop on Hyperspectral Image and Signal Processing: Evolution in Remote Sensing (IEEE), 2014.

[20] J. A. Griffiths, G. J. Royle, A. M. Hanby, J. A. Horrocks, S. E. Bohndiek, R. D. Speller, Correlation of energy dispersive diffraction signatures and microCT of small breast tissue samples with pathological analysis, Physics in Medicine and Biology 52 (2007) 6151-6164. 
[21] E. Ryan, M. Farquharson, Breast tissue classification using x-ray scattering measurements and multivariate data analysis, Physics in Medicine and Biology 52 (2007) 6679-6696.

[22] M. E. Poletti, O. D. Gonalves, I. Mazzaro, X-ray scattering from human breast tissues and breast-equivalent materials, Physics in Medicine and Biology 47 (2002) 47.

[23] C. R. F. Castro, R. C. Barroso, M. J. Anjos, R. T. Lopes, D. Braz, Coherent scattering characteristics of normal and pathological breast human tissues, Radiation Physics and Chemistry 71 (2004) 649-651.

[24] O. R. Oliveira, A. L. C. Conceição, D. M. Cunha, M. E. Poletti, C. A. Pelá, Identification of Neoplasias of Breast Tissues Using a Powder Diffractometer, Journal of Radiation Research 49 (2008) 527-532.

[25] A. Conceio, M. Antoniassi, M. Poletti, L. Caldas, Preliminary study of human breast tissue using synchrotron radiation combining WAXS and SAXS techniques, Applied Radiation and Isotopes 68 (2010) 799-803.

[26] D. L. Batchelar, Bone-composition imaging using coherent-scatter computed tomography: Assessing bone health beyond bone mineral density, Medical Physics 33 (2006) 904-915.

[27] G. Poludniowski, G. Landry, F. DeBlois, P. M. Evans, F. Verhaegen, SpekCalc: a program to calculate photon spectra from tungsten anode x-ray tubes, Physics in Medicine and Biology 54 (2009) N433.

[28] J. S. Francesc Salvat, Jos M. Fernndez-Varea, PENELOPE-2008: A Code System for Monte Carlo Simulation of Electron and Photon Transport, OECD, 2008.

[29] F. A. Duck, Physical Properties of Tissue: A Comprehensive Reference Book, Academic Press, 1990. 
[30] G. Harding, B. Schreiber, Coherent X-ray scatter imaging and its application in biomedical science and industry, Radiation Physics and Chemistry 56 (1999) 229-245.

[31] J. Greenberg, K. Krishnamurthy, D. Brady, Snapshot molecular imaging using coded energy-sensitive detection, Optics Express 21 (2013) 2548025491.

[32] M. N. Lakshmanan, R. E. Morris, J. A. Greenberg, E. Samei, A. J. Kapadia, Coded aperture coherent scatter imaging for breast cancer detection: a Monte Carlo evaluation, in: Proc. SPIE 9783, Medical Imaging 2016: Physics of Medical Imaging, 2016.

[33] G. Montemont, S. Lux, O. Monnet, S. Stanchina, L. Verger, Studying Spatial Resolution of CZT Detectors Using Sub-Pixel Positioning for SPECT, IEEE Transactions on Nuclear Science 61 (2014) 2559-2566.

[34] A. Michalowska, O. Gevin, O. Lemaire, F. Lugiez, P. Baron, H. Grabas, F. Pinsard, O. Limousin, E. Delagnes, IDeF-X HD: A low power multi-gain CMOS ASIC for the readout of Cd(Zn)Te detectors, in: 2010 IEEE Nuclear Science Symposium Conference Record (NSS/MIC), 2010.

[35] G. Montemont, M.-C. Gentet, O. Monnet, J. Rustique, L. Verger, Simulation and Design of Orthogonal Capacitive Strip CdZnTe Detectors, in: IEEE Nuclear Science Symposium Conference Record, 2006, 2006.

[36] I. A. Cunningham, R. Shaw, Signal-to-noise optimization of medical imaging systems, Journal of the Optical Society of America A, Optics and Image Science 16 (1999) 621-632.

[37] S. ke Starck, M. Bth, S. Carlsson, The use of detective quantum efficiency (DQE) in evaluating the performance of gamma camera systems, Physics in Medicine and Biology 50 (2005) 1601-1609. 
[38] J. A. Greenberg, M. N. Lakshmanan, D. J. Brady, A. J. Kapadia, Optimization of a coded aperture coherent scatter spectral imaging system for medical imaging, in: Proc. SPIE, 2015.

[39] D. J. Rhodes, M. K. O'Connor, S. W. Phillips, R. L. Smith, D. A. Collins, Molecular Breast Imaging: A New Technique Using Technetium Tc 99m Scintimammography to Detect Small Tumors of the Breast, Mayo Clinic Proceedings 80 (2005) 24-30.

[40] M. K. O'Connor, S. W. Phillips, C. B. Hruska, D. J. Rhodes, D. A. Collins, Molecular breast imaging: Advantages and limitations of a scintimammographic technique in patients with small breast tumors, The Breast Journal 13 (2007) 3-11.

[41] L. Shepp, Y. Vardi, Maximum likelihood reconstruction for emission tomography, IEEE Transactions on Medical Imaging 1 (1982) 113-122.

[42] F. Marticke, G. Montémont, C. Paulus, O. Michel, J. I. Mars, L. Verger, Calcul analytique de courbes COR en imagerie de diffraction X, in: Gretsi, 2015.

[43] R. A. Parry, S. A. Glaze, B. R. Archer, The aapm/rsna physics tutorial for residents: Typical patient radiation doses in diagnostic radiology, RadioGraphics 19 (1999) 1289-302.

[44] D. Hintenlang, Dose measurement in mammography; what are we measuring?, in: ACMP Annual Meeting, 2008.

[45] T. Olgar, T. Kahn, D. Gosch, Average glandular dose in digital mammography and breast tomosynthesis, RöFo - Fortschritte auf dem Gebiet der Röntgenstrahlen und der bildgebenden Verfahren 184 (2012) 911-918. 